\title{
Excess Volume of Binary Mixtures of Water (Methanol or Ethanol) with an Ionic Liquid [EEIM][DEP] or [BEIM][DEP] at 1 atm and (293.15 to 333.15$) \mathrm{K}$
}

\author{
Hong-Yan Song ${ }^{\mathrm{a}}$, Yin-Hui Gong ${ }^{\mathrm{b}}$, Xue-Mei Jiang ${ }^{\mathrm{b}, \mathrm{c}}$ and Chun-Xi Li, ${ }^{\mathrm{b},}$ \\ ${ }^{a}$ Faculty of Science, Beijing University of Chemical Technology, Beijing 100029, China \\ ${ }^{b}$ College of Chemical Engineering, Beijing University of Chemical Technology, Beijing 100029, China \\ ${ }^{c}$ China Petroleum Engineering Co. Ltd., Beijing 100085, China
}

\begin{abstract}
Densities were measured for the binary mixtures of water (methanol or ethanol) and an ionic liquid (IL) 1,3diethylimidazolium diethylphosphate [EEIM][DEP] or 1-n-butyl-3-ethylimidazolium diethylphosphate [BEIM][DEP] at 1 atm and (293.15 to 333.15$) \mathrm{K}$ using a vibrating-tube densimeter. The molecular volume, standard entropy, and lattice energy of the two ILs were estimated with the Glasser theory. The excess volumes are negative for all binary mixtures studied in the whole composition range, and reach to the maximum at the mole fraction of IL being around 0.3 . Besides, they decrease with increasing temperature for the aqueous solution of ILs, whilst a reverse trend is found for the IL solutions of methanol or ethanol at any concentration. The excess molar volumes were correlated successfully by a fiveparameter polynomial equation as a function of temperature and mole fraction of IL with average absolute relative deviation (ARD) of density within $0.02 \%$.
\end{abstract}

Keywords: Density, excess volume, ionic liquid, water, methanol, ethanol.

\section{INTRODUCTION}

Ionic liquids (ILs), as a novel class of organic molten salts at ambient temperatures, show the characteristics of both electrolyte and non-electrolyte. And their ionic attribute is highly dependent on the solvent property and interaction with the solvent, giving rise to varying non-ideality of solution behavior. To reveal the nonideality of the IL solutions from the aspect of excess volume, density property is measured for binary ILs solutions at different conditions. As a continuation study on the thermodynamic properties of ILs [1-3], we measured the density of binary mixtures of water (methanol or ethanol) and an IL ([EEIM][DEP] or [BEIM][DEP]) at $1 \mathrm{~atm}$ in the temperature range of (293.15 to 333.15$) \mathrm{K}$. The volume properties of the ILs were discussed in terms of the Glasser's theory. The excess molar volumes $\left(V^{E}\right)$ are correlated as a function of temperature and composition by an empirical polynomial equation. The solution behavior of these ILcontaining mixtures was discussed in terms of the variation of excess molar volume with temperature, composition and the constituents of the mixtures.

\section{EXPERIMENTAL}

\subsection{Materials}

The chemicals used are $\mathrm{N}$-ethylimidazole and $\mathrm{N}$ butylimidazole ( $\geq 99.5$ wt. $\%$, Zhejiang Kaile Reagents

*Address correspondence to this author at the College of Chemical Engineering, Beijing University of Chemical Technology, Beijing 100029, China; Tel/Fax: 86-10-64410308; E-mail: Licx@mail.buct.edu.cn
Company, China), triethylphosphate ( $\geq 99.5$ wt.\%, Tianjin Guangfu Reagents Company, China) and aether ( $\geq 99.5$ wt.\%, Beijing Yili Reagents Company, China), which were used as received. Methanol and ethanol were supplied by Beijing Chemical Plant with nominal purity above 99.7 wt.\%, degassed ultrasonically before use, and stored over the activated fresh molecular sieves of type $4 \AA$ (Union Carbide) in tightly sealed glass bottles. The ILs [EEIM][DEP] and [BEIM][DEP] were synthesized and purified in our laboratory according to the method described in the literature [4], and their purities are better than $99 \mathrm{wt} . \%$ as determined by the NMR analysis. Water content in the ILs is below $0.032 \%$ as determined by the Karl Fisher titrator (type CBS-1A).

\subsection{APPARATUS AND PROCEDURE}

The densities of pure ILs and their binary mixtures with water (methanol or ethanol) at different temperatures were measured using the digital vibrating tube densimeter (DMA 4500M, Anton Paar Co. Ltd., Austria). The binary mixtures with different mole fractions of ILs were prepared by weighting with an electronic balance (type AR 2130, Ohaus Co. Ltd., USA) with a mass precision of $\pm 0.001 \mathrm{~g}$. The nominal uncertainty of the experimental density is $\pm 1 \times 10^{-5}$ $\mathrm{g} \cdot \mathrm{cm}^{-3}$. The temperature of the densimeter measuring cell is better than $0.002 \mathrm{~K}$ and the precision of measurement is $\pm 0.01 \mathrm{~K}$ with a built-in thermostat. The accuracy of the density data is verified by comparing the experimental data of water with the reference 
Table 1: Density $\rho /\left(\mathrm{g} \cdot \mathrm{cm}^{-3}\right)$ of Pure ILs at $1 \mathrm{~atm}$ and (293.15 to 333.15) K

\begin{tabular}{|c|c|c|c|c|c|c|c|c|c|}
\hline \multirow{2}{*}{ Ionic liquid } & \multicolumn{9}{|c|}{$T / K$} \\
\hline & 293.15 & 298.15 & 303.15 & 308.15 & 313.15 & 318.15 & 323.15 & 328.15 & 333.15 \\
\hline [EEIM][DEP] & 1.12932 & 1.12596 & 1.12260 & 1.11924 & 1.11588 & 1.11251 & 1.10915 & 1.10580 & 1.10247 \\
\hline [BEIM][DEP] & 1.08660 & 1.08321 & 1.07984 & 1.07650 & 1.07315 & 1.06980 & 1.06645 & 1.06310 & 1.05976 \\
\hline
\end{tabular}

values [5] from 293.15 to 333.15 with the average relative deviation within $0.02 \%$.

\section{RESULTS AND DISCUSSION}

\subsection{Density and Derived Properties of Pure ILs}

The densities of pure ILs [EEIM][DEP] and [BEIM][DEP] at $1 \mathrm{~atm}$ were measured in the temperature range of (293.15 to 333.15$) \mathrm{K}$, and listed in Table 1. The density of the pure components studied here follows the order of ethanol $\approx$ methanol<water $<[B E I M][D E P]<\quad[E E I M][D E P]$ in the fixed other conditions. The density of ILs is generally higher than that of molecular solvents due to the strong electrostatic interaction among ions of the ILs. Besides, the density of ILs decreases with the increase of the length of the alkyl substitute to the imidazolium ring, implying that the electrostatic interaction plays a predominant role since a shorter alkyl substitute means a smaller ionic size and a lower steric hinderance for the closest approaches among ionic species and thus a stronger Columbic interaction albeit the dispersion interaction is a little weaker for the ILs with shorter alkyl substitutes.

Density results for the pure ILs were used to derive other thermodynamic properties such as the coefficient of thermal expansion, molar volume, standard entropy and lattice energy. The coefficient of thermal expansion $\alpha$ of a liquid is defined by the following equation:

$\alpha=\frac{1}{V}\left(\frac{\partial V}{\partial T}\right)_{\mathrm{P}}=-\left(\frac{\partial \ln \rho}{\partial T}\right)_{\mathrm{p}}$

where $V$ and $\rho$ is the molar volume and density of the IL, respectively. The variation trend of In $\rho$ vs. $T$ for the two ILs studied is presented in Figure 1. As shown in the figure, the values of In $\rho$ decrease slightly in a linear way with the increasing temperature. The $\alpha$ values were obtained from the slope of the linear regression of $\operatorname{In} \rho$ against $T$, being about $6.02 \times 10^{-4} \mathrm{~K}^{-1}$ and $6.25 \times 10^{-4} \mathrm{~K}^{-1}$, respectively, for [EEIM][DEP] and [BEIM][DEP]. The coefficient of thermal expansion of the ILs is quite low, and increases with the length of the alkyl substitutes to the imidazolium ring as a result of the deceasing electrostatic interaction among ionic species in the ILs involved.

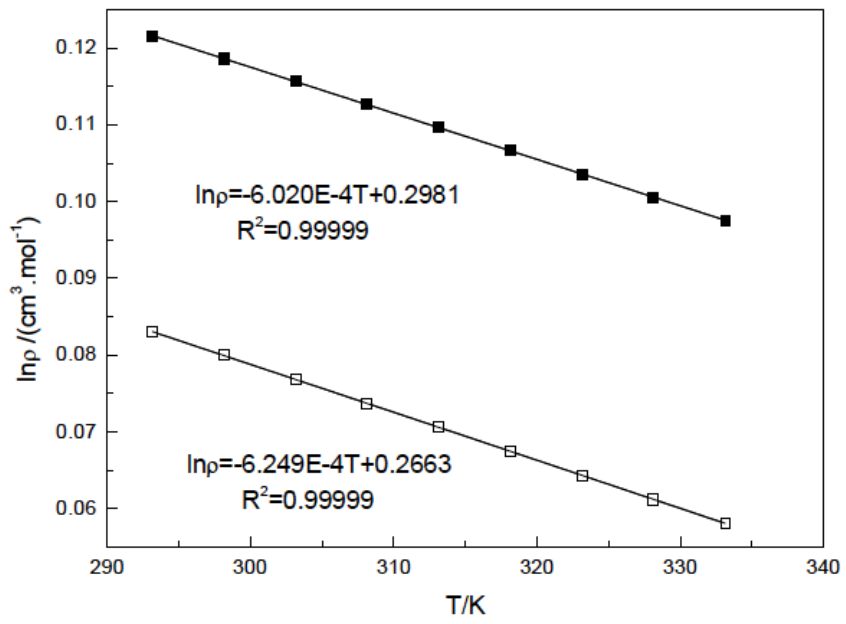

Figure 1: Plot of $\ln \left(\rho / \mathrm{g} \cdot \mathrm{cm}^{-3}\right)$ against $T / \mathrm{K}$ for ILs: (匹), [EEIM][DEP]; (), [BEIM][DEP].

From the experimental density data, the molecular volume of the ILs, $V_{\mathrm{m}}$, can be estimated using the following equation.

$V_{\mathrm{m}}=M /\left(N_{\mathrm{A}} \cdot \rho\right)$

where $M$ is the molar mass, $N_{\mathrm{A}}$ is the Avogadro constant, $\rho$ is the density. The $V_{\mathrm{m}}$ values thus determined for [EEIM][DEP] and [BEIM][DEP] at $298.15 \mathrm{~K}$ are 0.4106 and $0.4698 \mathrm{~nm}^{3}$, respectively. By comparing the $V_{\mathrm{m}}$ values of these ILs, the mean contribution of a methylene group $\left(-\mathrm{CH}_{2}-\right)$ to the molecular volume is about $0.0289 \mathrm{~nm}^{3}$, which is close to the literature value $0.0293 \mathrm{~nm}^{3}$ derived from ILs [MMIM][DMP] and [EMIM][DEP] [6], $0.0280 \mathrm{~nm}^{3}$ from $\mathrm{n}$-alcohols, $0.0272 \mathrm{~nm}^{3}$ from $\mathrm{n}$-amines and $0.0267 \mathrm{~nm}^{3}$ from n-paraffins [7].

The standard entropy, $S^{\circ}$, can be calculated in terms of the molecular volume and a good linear relationship proposed by Glasser [7]. The linear correlation constants in Eq. (3) are appropriate for the ILs with large organic cations. 
Table 2: The Molecular Volume, $V_{\mathrm{m}}$, Standard Entropy, $S^{\circ}$, and Lattice Energy, $U_{\mathrm{POT}}$, Derived from Density of [EEIM][DEP] and [BEIM][DEP] at 298.15 K

\begin{tabular}{|c|c|c|c|c|c|}
\hline Ionic liquid & $\mathbf{M} /\left(\mathbf{g} \cdot \mathbf{m o l}^{-1}\right)$ & $\boldsymbol{\rho} /\left(\mathbf{g} \cdot \mathbf{c m}^{-3}\right)$ & $\boldsymbol{V}_{\mathbf{m}} /\left(\mathbf{n m} \mathbf{m}^{3}\right)$ & $\boldsymbol{S}^{\mathbf{0}} /\left(\mathbf{J} \cdot \mathbf{m o l}^{-1} \cdot \mathbf{K}^{-1}\right)$ & $\boldsymbol{U}_{\mathrm{POT}} /(\mathbf{k J} \cdot \mathbf{m o l}$ \\
\hline \hline$[E E I M][D E P]$ & 278.29 & 1.12596 & 0.4106 & 541.3 & 419.5 \\
\hline$[B E I M][D E P]$ & 306.34 & 1.08321 & 0.4698 & 615.1 & 405.6 \\
\hline
\end{tabular}

$S^{0}(298) /\left(\mathrm{J} \cdot \mathrm{mol}^{-1} \cdot \mathrm{K}^{-1}\right)=1246.5\left(V_{\mathrm{m}} / \mathrm{nm}^{3}\right)+29.5$

The $S^{\circ}$ values calculated by Eq. (3) for the two ILs are listed in Table 2, from which the mean entropy contribution per methylene group $\left(-\mathrm{CH}_{2^{-}}\right)$to the standard entropy is determined as $36.0 \mathrm{~J} \cdot \mathrm{mol}^{-1} \cdot \mathrm{K}^{-1}$. This is comparable to the literature value $36.5 \mathrm{~J} \cdot \mathrm{mol}^{-}$ ${ }^{1} \cdot \mathrm{K}^{-1}$ derived from ILs [MMIM][DMP] and [EMIM][DEP] [6], $35.1 \mathrm{~J} \cdot \mathrm{mol}^{-1} \cdot \mathrm{K}^{-1}$ from $\left[\mathrm{C}_{\mathrm{n}} \mathrm{mim}\right]\left[\mathrm{NTf}_{2}\right], 33.9 \mathrm{~J} \cdot \mathrm{mol}^{-1} \cdot \mathrm{K}^{-1}$ from $\left[\mathrm{C}_{\mathrm{n}} \mathrm{mim}\right]\left[\mathrm{BF}_{4}\right][7]$ and $34.1 \mathrm{~J} \cdot \mathrm{mol}^{-1} \cdot \mathrm{K}^{-1}$ from $\left[\mathrm{C}_{\mathrm{n}} \mathrm{mim}\right]\left[\mathrm{AlCl}_{4}\right][8]$.

Density data is also useful for the estimation of lattice energy ( $U_{\text {РОт }}$ ) of ILs. According to the Glasser's theory, the lattice energy depends only on the chemical formula, ionic charges and molecular volume of the materials involved, and neither relies on any other structural information. For the present case of ionic liquids of $\mathrm{MX}$ type with charge ratio 1:1, the Columbic interaction is the principal contribution to the lattice energy, and the $U_{\text {рот }}$ values can be estimated by Eq. (4).

$U_{\text {POT }} /\left(\mathrm{kJ} \cdot \mathrm{mol}^{-1}\right)=1981.2(\rho / M)^{1 / 3}+103.8$

As shown in Table 2, the lattice energies thus determined for [EEIM][DEP] and [BEIM][DEP] are $419.5 \mathrm{~kJ} \cdot \mathrm{mol}^{-1}$ and $405.6 \mathrm{~kJ} \cdot \mathrm{mol}^{-1}$, respectively. Obviously, the lattice energy of ILs is much lower than that of the fused inorganic salts, for example, the lattice energy of fused CsI which has the least crystal energy among all the alkali halides is about $602.5 \mathrm{~kJ} \cdot \mathrm{mol}^{-1}$ [9]. The low lattice energy may be the underlying reason for forming ILs at room temperature [8].

\subsection{Density of Binary Mixtures}

The density data for the binary mixtures at the mole fraction of $\mathrm{IL}, x_{\mathrm{IL}}$, being about $0.2,0.4,0.6$, and 0.8 , were measured at the same pressure and temperature as that for the pure components, and listed in Table 3.

\subsection{Excess Molar Volume of Binary Mixtures}

The excess molar volume, $V^{E}$, is an important thermodynamic property to represent the non-ideality of a solution, which is defined as the difference of the molar volume between the real mixture $\left(v_{\mathrm{m}}\right)$ and the ideal solution $\left(v_{\mathrm{m}, \mathrm{id}}\right)$ at the same temperature, pressure and composition i.e.

$$
V^{\mathrm{E}}=V_{\mathrm{m}}-V_{\mathrm{m}, \mathrm{id}}
$$

The $V_{\mathrm{m}}$ and $V_{\mathrm{m}, \mathrm{id}}$ values can be calculated as follows:

$$
\begin{gathered}
V_{\mathrm{m}}=\frac{x_{\mathrm{IL}} M_{\mathrm{IL}}+x_{\mathrm{S}} M_{\mathrm{S}}}{\rho_{\text {mix }}} \\
V_{\mathrm{m}, \mathrm{id}}=\frac{x_{\mathrm{IL}} M_{\mathrm{IL}}}{\rho_{\mathrm{IL}}}+\frac{x_{\mathrm{S}} M_{\mathrm{S}}}{\rho_{\mathrm{S}}}
\end{gathered}
$$

where $M_{\mathrm{S}}$ and $M_{\mathrm{IL}}$ are the mole masses of solvent and $\mathrm{IL}$, respectively. $x_{S}$ and $x_{\mathrm{IL}}$ represent the mole fractions of solvent and $\mathrm{IL}$, respectively. $\rho_{\mathrm{S}}, \rho_{\mathrm{IL}}$ and $\rho_{\text {mix }}$ refer to the densities of solvent, IL and the mixture, respectively.

Based on the experimental data of density, as listed in Tables 1 and 3, the excess molar volume for the binary mixtures at different conditions were calculated by Eqs. 5 to 7. The results show that the excess volumes are negative for all the systems studied at any temperature and concentration, indicating a negative deviation from the ideal solution behavior. Besides, the excess volume is relatively low being in the range of $(0.9$ to 1.9$) \mathrm{cm}^{3} \cdot \mathrm{mol}^{-1}$ for all the binary mixtures studied, and reaches the maximum at the mole fraction of IL being around 0.3 . For the convenience of illustration, plots of excess molar volume against the mole fraction of IL at different temperatures for the binary mixtures of [EEIM][DEP] with ethanol and water are presented in Figures 2 and 3, respectively. As shown in Figure 2, the excess molar volume of the binary mixture $([E E I M][D E P]+$ ethanol) increases with increasing temperature at fixed other conditions. This trend holds for all binary mixtures of (IL+ alcohols) studied here and in good agreement with those reported elsewhere for other ILs mixtures with different alcohols [10, 11]. 
Table 3: Density Data Measured for the Binary Mixtures at $1 \mathrm{~atm}$, Different Temperatures and Mole Fractions of ILs

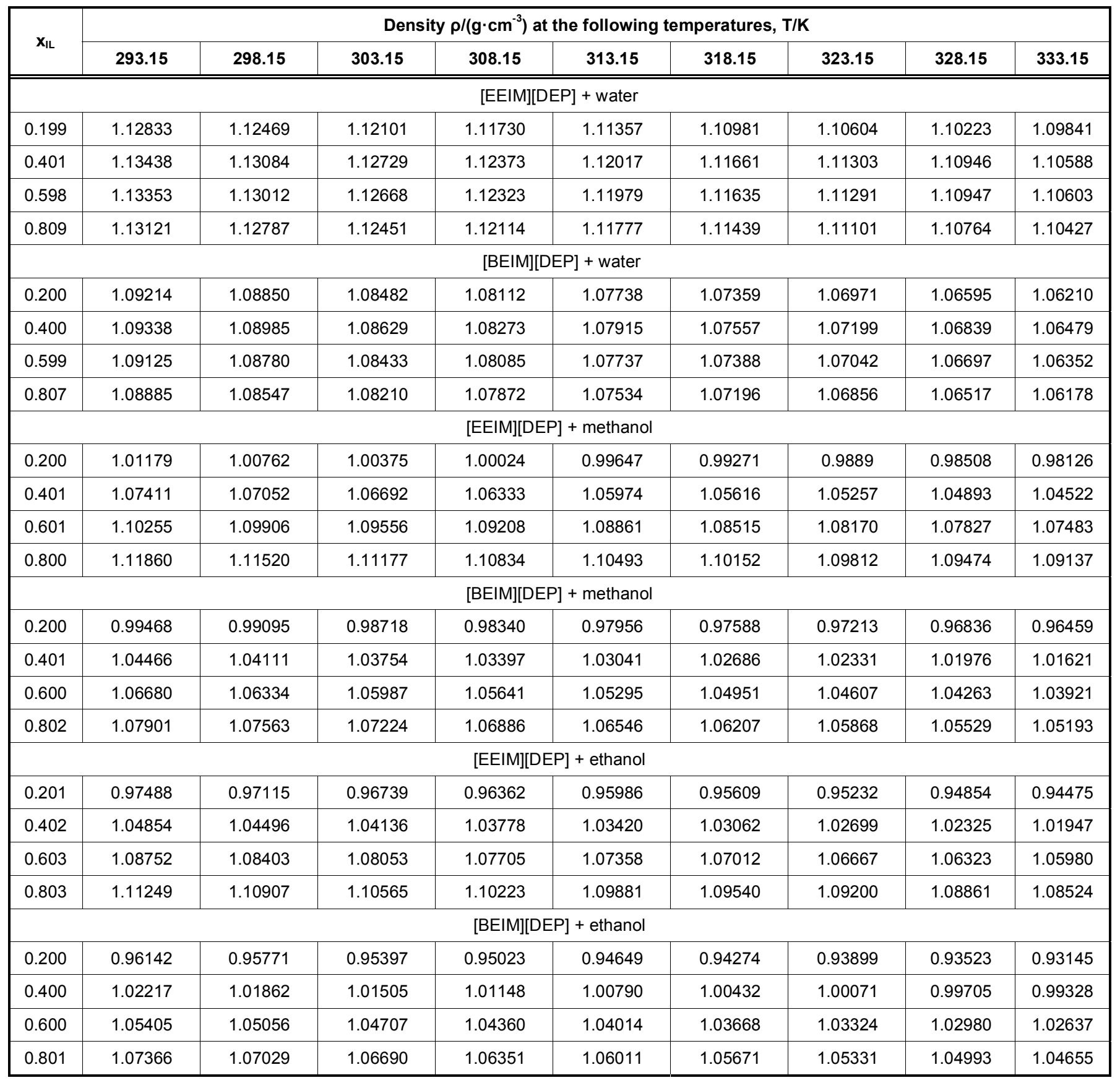

In contrast to the binary mixtures of IL plus alcohols, the excess volumes of the (IL + water) mixtures decrease as the temperature increasing, as shown in Figure 3, implying that the non-ideality of the mixture is higher at lower temperature and decreases as the temperature increasing. This variation trend may be attributed to the strong dielectric property of water and its decreasing dielectric behavior as temperature increasing, for example, the dielectric constant of water decreases from 80.1 at $293 \mathrm{~K}$ to 63.8 at $333 \mathrm{~K}$ [5]. The high dielectric property of water makes the ionic liquid partially dissociated into ions, leading to a high asymmetrical interaction between neutral water molecules and charged particles of the IL component, and accordingly a high non-ideality of the mixture and the excess volumes. This means that an aqueous solution of ILs usually shows a larger deviation from the ideal solution behavior than an alcoholic solution of ILs, which is justified by the fact that the values of $V^{E}$ for the (IL + water) mixtures are always larger than those for 
the $(\mathrm{IL}+$ alcohol) mixtures at the same temperature and concentration. Besides, as the temperature increases the ILs component is apt to present as neutral molecules rather than as ionic species due to the decreasing dielectric constant of water, leading to a lower non-ideality and excess volume.

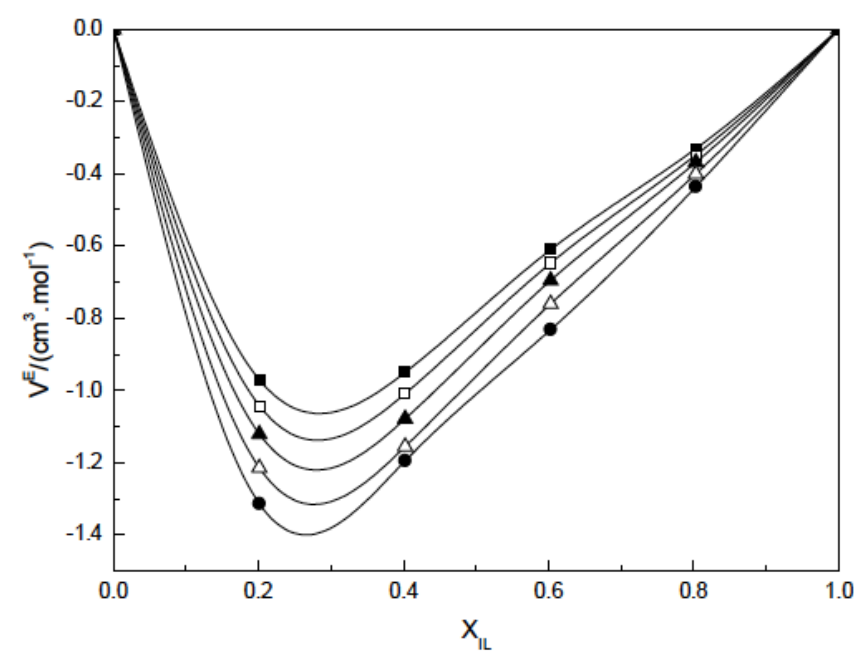

Figure 2: The experimental and correlative excess molar volume for the binary mixture ([EEIM][DEP] + ethanol) at different mole fractions of IL and temperatures: (-), $293.15 \mathrm{~K}$; (), $303.15 \mathrm{~K} ;(\bullet), 313.15 \mathrm{~K} ;(\Delta), 323.15 \mathrm{~K} ;(\bullet), 333.15 \mathrm{~K}$; (一), calculated values from Eq. (8).

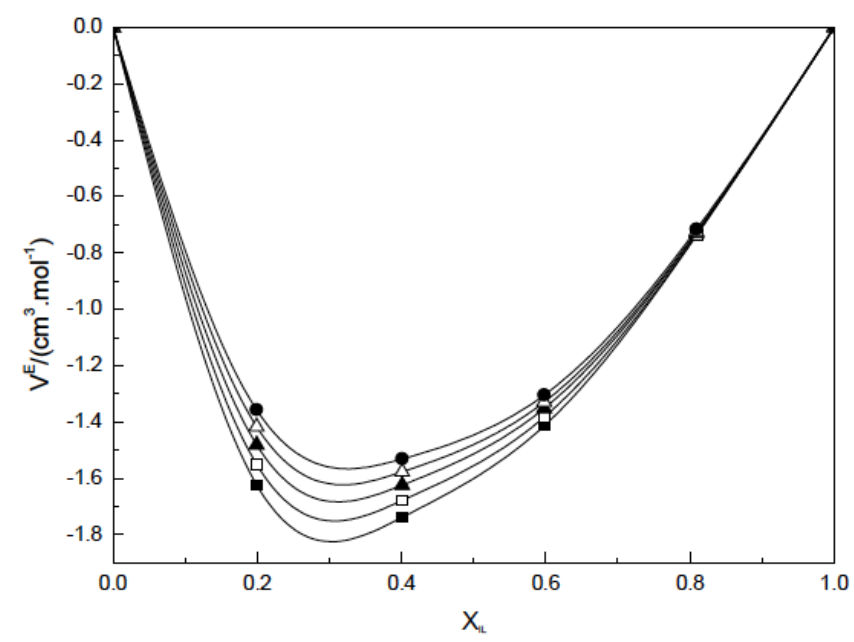

Figure 3: The experimental and correlative excess molar volume for the binary mixture ([EEIM][DEP] + water) at different mole fractions of IL and temperatures: $(\bullet), 293.15 \mathrm{~K}$; $(\Delta), 303.15 \mathrm{~K} ;(\boldsymbol{\Delta}), 313.15 \mathrm{~K}$; (), $323.15 \mathrm{~K} ;(\bullet), 333.15 \mathrm{~K}$; $(-)$, calculated values from Eq. (8).

For a specific ionic liquid, the solvent property has an important influence on the non-ideality and excess molar volume of the IL-containing binary solutions, as an illustrative example, Figure $\mathbf{4}$ presents the excess molar volumes against the mole fraction of IL for the binary mixtures of [EEIM][DEP] with water, methanol and ethanol at $298.15 \mathrm{~K}$. It is seen that among the three binary mixtures, the aqueous solution of IL shows the highest excess molar volume and the ethanol solution of IL shows the lowest one at the same temperature and mole fraction of IL. This trend is in agreement with the dielectric constant [5] order at $298.15 \mathrm{~K}$, viz. water (78.3) > methanol (32.7) > ethanol (24.5). As a result, the aqueous solution of IL is similar to an electrolyte solution, while the alcoholic solution of IL is more like a non-electrolytic organic mixture. In effect, the excess volume is a reflection of the compromising effects among molecular sizes and various interactions for a specific mixture, and is dependent on the temperature and composition. The negative excess volume may be associated with the strong ion-dipole and dipole-dipole interactions between IL and the solvent molecules, as well as the interstitial effect in molecular packing due to the differences in the size and shape of the component molecules, by which the volume of the liquid mixture is apt to be contracted.

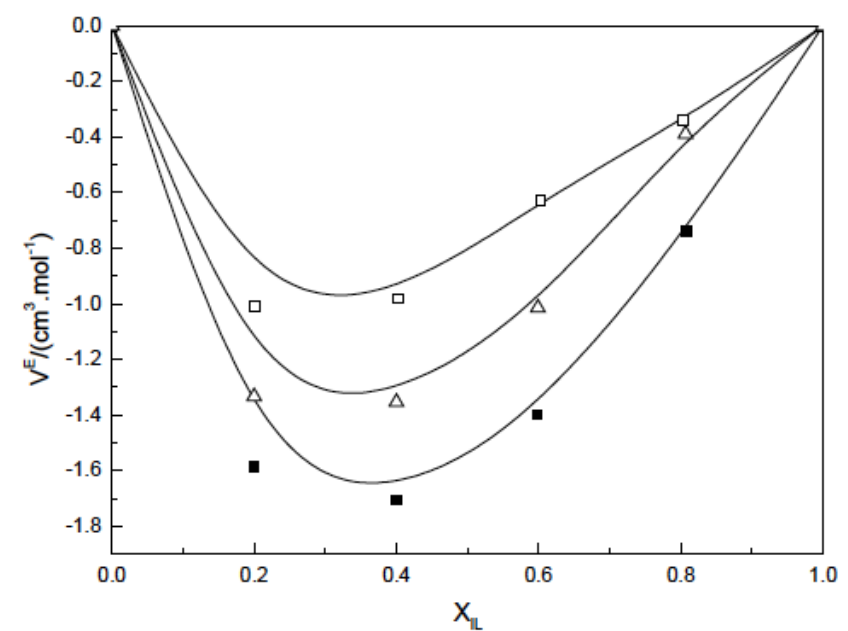

Figure 4: Excess molar volume for the binary mixtures of [EEIM][DEP] with different solvents at 298.15K: (口), [EEIM][DEP] + ethanol; $(\Delta)$, [EEIM][DEP] + methanol;. (匹), $[E E I M][D E P]+$ water.

\subsection{Correlation of the Density Data}

In view of the complex variation of the excess molar volume with temperature and IL-content for a binary mixture, the experimental values of $V^{E}$ are fitted to the following equation (8) by the least-square method.

$$
V^{\mathrm{E}}=x_{\mathrm{IL}}\left(1-x_{\mathrm{IL}}\right) \sum_{\mathrm{i}=0}^{\mathrm{j}} A_{\mathrm{i}}\left(1-2 x_{\mathrm{IL}}\right)^{\mathrm{i}}\left(1+B T+C T^{2}\right)
$$

Where $x_{\mid \mathrm{L}}$ is the mole fraction of IL, $T$ is the absolute temperature in $\mathrm{K}$. The best fit was obtained when five adjustable parameters were used in Eq. 8. The resulting parameters $A_{0}, A_{1}, A_{2}, B, C$, and the average 
Table 4: The Regressed Parameters of Eq. (8) Along with the Deviation in ARD for the Binary Systems

\begin{tabular}{|c|c|c|c|}
\hline $\begin{array}{l}\text { Correlation results } \\
\text { [EEIM][DEP] IL }\end{array}$ & \multicolumn{3}{|c|}{ IL + solvents binary systems } \\
\hline$A_{0}$ & -0.00079 & -0.00032 & -0.00034 \\
\hline$A_{1}$ & -0.00047 & -0.00030 & -0.00037 \\
\hline$A_{2}$ & -0.00033 & -0.00020 & -0.00028 \\
\hline C & -0.15506 & 0.12047 & 0.13907 \\
\hline $\operatorname{ARD}\left(V^{E}\right)$ & $1.5930 \%$ & $1.7324 \%$ & $0.7284 \%$ \\
\hline $\operatorname{ARD}(\rho)$ & $0.0175 \%$ & $0.0146 \%$ & $0.0043 \%$ \\
\hline [BEIM][DEP] IL & $\mathrm{IL}+$ water & IL + methanol & IL + ethanol \\
\hline$B$ & 26.54501 & 12.87334 & -4.51104 \\
\hline C & -0.05934 & 0.06443 & 0.08369 \\
\hline $\operatorname{ARD}\left(V^{E}\right)$ & $1.1104 \%$ & $2.4621 \%$ & $2.9749 \%$ \\
\hline $\operatorname{ARD}(\rho)$ & $0.0104 \%$ & $0.0182 \%$ & $0.0129 \%$ \\
\hline
\end{tabular}

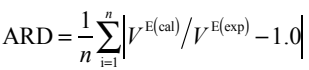

relative deviations of excess molar volume between the experimental and correlated values, ARD $\left(V^{E}\right)$, for each binary system are listed in Table 4 . The experimental density data were accurately correlated by using Eqs. (5) through (8) with the highest correlative deviation in terms of $\operatorname{ARD}(\rho)$ within $0.02 \%$, which is close to the experimental uncertainty of the densimeter used here. The goodness of Eqs. (5) through (8) for representing density and excess molar volume of the IL-containing binary mixtures can be clearly seen from Figures 2 through $\mathbf{4}$, as well as the very low ARD values as listed in Table 4.

\section{CONCLUSIONS}

The density data for the binary mixtures of an IL of [EEIM][DEP] or [BEIM][DEP], and a solvent of water, methanol or ethanol, were measured at $1 \mathrm{~atm}$ in the temperature range of (293.15 to 333.15$) \mathrm{K}$ using densimeter. The excess volumes are all negative in the whole temperature and concentration range studied, indicating a negative deviation from the ideality, and the variation of excess volume with temperature shows two opposite trends for the (IL + water) mixtures and the (IL + alcohol) mixtures. Moreover, the excess volume as well as the density data can be correlated accurately by a five-parameter empirical polynomial equation.

\section{ACKNOWLEDGEMENTS}

The authors are grateful to the financial support from National Natural Science Foundation of China (21076005).

\section{REFERENCES}

[1] Gong Y, Lu Y, Meng H, Li C. Density measurement for the binary mixtures of ionic liquid 1-ethyl-3-methylimidazolium diethylphosphate and water(methanol or ethanol) at $1 \mathrm{~atm}$ and (293.15 to 333.15) K. Journal of Applied Solution Chemistry and Modeling 2013; 2: 234-9.

[2] Wang J, Li Z, Li C, Wang Z. Density prediction of ionic liquids using a group contribution equation of state. Ind Eng Chem Res 2010; 49: 4420-5. http://dx.doi.org/10.1021/ie901590h

[3] Shen C, Li C, Li X, Lu Y. Muhammad Y. Estimation of densities of ionic liquids using Patel-Teja equation of state and critical parameters determined from group contribution method. Chem Eng Sci 2011; 66: 2690-8. http://dx.doi.org/10.1016/j.ces.2011.03.027

[4] Zhou Y, Robertson AJ, Hillhouse JH, Baumann D. Phosphonium and imidazolium salts and methods of their preparation. WO 2004/016631 Al, 26 Feb., 2004.

[5] Liu G, Ma L, Liu J. Physical property for chemistry and chemical engineering, 1st ed., Chemical Industry Press, Beijing, 2002. 
[6] Wang J, Zhao F, Liu R, Hu Y. Thermophysical properties of 1-methyl-3-methylimidazolium dimethylphosphate and 1ethyl-3-methylimidazolium diethylphosphate. J Chem Thermodyn 2011; 43: 47-50.

http://dx.doi.org/10.1016/j.jct.2010.08.002

[7] Glasser L. Lattice and phase transition thermodynamics of ionic liquids. Thermochim Acta 2004; 421: 87-93. http://dx.doi.org/10.1016/.tca.2004.03.015

[8] Tong J, Hong M, Guan W, Li JB, Yang JZ. Studies on the thermodynamic properties of new ionic liquids: 1-Methyl-3pentylimidazolium salts containing metal of group III. J Chem Thermodyn 2006; 38: 1416-21.

http://dx.doi.org/10.1016/j.jct.2006.01.017
[9]

Huang ZQ. The Introduction of Electrolyte Solution Theory, divided ed., Science Press, Beijing, 1983, p. 98.

[10] Bhuiyan $\mathrm{MMH}$, Uddin $\mathrm{MH}$. Excess molar volumes and excess viscosities for mixtures of $\mathrm{N}, \mathrm{N}$-dimethylformamide with methanol, ethanol and 2-propanol at different temperatures. Journal of Molecular Liquids 2008; 138: 139-46. http://dx.doi.org/10.1016/j.molliq.2007.07.006

[11] Hofman T, Goldon A, Nevines A, Letcher TM. Densities, excess volumes, isobaric expansibity, and isothermal compressibility of the (1-ethyl-3-methylimidazolium ethylsulfate + methanol) system at temperatures (283.15 to 333.15) $\mathrm{K}$ and pressures from (0.1 to 35$) \mathrm{MPa}$. J Chem Thermodyn 2008; 40: 580-91. 